\title{
Efficacy and Safety of tDCS and tACS in Treatment of Major Depressive Disorder: A Randomized, Double-Blind, Factorial Placebo-Controlled Study Design
}

\author{
Yuxin Huang' \\ Linjie Shen ${ }^{\prime}{ }^{\prime}$ \\ Jia Huang' \\ Xianrong $\mathrm{Xu}^{2}$ \\ Yong Wang' \\ Hua Jin' \\ 'Department of Outpatient, Shanghai \\ Mental Health Center, Shanghai, People's \\ Republic of China; ${ }^{2}$ School of Public \\ Health, Hangzhou Normal University, \\ Hnagzhou, Zhejiang Province, People's \\ Republic of China
}

Correspondence: Yong Wang Department of Outpatient, Shanghai Mental Health Center, No. 600 Wanping South Road, Xuhui District, Shanghai,

People's Republic of China

Tel +86-180173II 332

Email tianlai1977@yeah.net
Background: Transcranial direct current stimulation (tDCS) and transcranial alternating current stimulation (tACS) are regarded as promising antidepressant treatments.

Objective: To compare the efficacy and safety of tDCS, tACS, escitalopram, and placebo/ sham stimulation controls.

Design: Randomized, parallel, double-blind, placebo-controlled study.

Methods: Sample sizes were calculated based on data from previous similar studies. Eligible non-treatment-resistant-depressive outpatient subjects with moderate-to-severe depression (HRDS $\geq 17$ ) are randomized to receive (1) tDCS + placebo; (2) tACS + placebo; (3) escitalopram + placebo; or (4) sham stimulation + placebo. The intensity of electricity is $2 \mathrm{~mA}$, lasting for 30 minutes over two consecutive working days (10 sessions in total). The medication lasts for 6 weeks. The primary outcome measure was the response rates within 6 weeks (week 6 is also the endpoint of the study), and secondary outcome measures included changes in other clinical measurements. Safety and acceptability are measured by adverse event rates and dropout rates. Exploring outcome consist of the performance of cognitive battery as well as neurophysiology results.

Conclusion: To the best of our knowledge, the present study is the first double-blind controlled study comparing tDCS, tACS, and clinically used antidepressants, which will provide further evidence for their efficacy and safety in possible clinical applications.

Keywords: transcranial direct current stimulation, tDCS, transcranial alternating current stimulation, tACS, electrical stimulation, major depressive disorder, MDD

\section{Introduction}

Major depressive disorder (MDD) is a widespread neuropsychiatric disorder with an estimated 12-month prevalence of $2.1 \%$ and a lifetime prevalence of $3.4 \%$ in China $^{1}$. Currently, the predominant treatment for MDD is antidepressant drugs, which only brings about $1 / 3$ clinical remission rate. ${ }^{2,3}$ Meanwhile, antidepressant drugs usually result in undesirable side effects and undergo 2-4 weeks to achieve clinical efficacy. Therefore, it is necessary to explore other alternative efficient approaches.

Non-invasive physical therapy interventions, including transcranial direct current stimulation (tDCS) and transcranial alternating current stimulation (tACS), have attracted the researchers' attention. Both tDCS and tACS stimulate the brain 
through scalp electrodes to modulate cortical activity, whose benefits include painlessness, causing few adverse events, portable, easy to operate, and economic.

\section{Transcranial Direct Current Stimulation (tDCS)}

TDCS delivers direct currents $(0.5-2 \mathrm{~mA})$ to the scalp to modulate the neural activity of the cerebrum. ${ }^{4,5}$ Its exact mechanism has yet to be defined. ${ }^{5}$ The stimulatory effect of tDCS is characterized by polarity regulation: the anode current promotes depolarization of neuronal membrane potentials to enhance cortical excitability, and the cathode current induces hyperpolarization changes to inhibit cortical excitability. ${ }^{6}$

TDCS potentially improves cognitive performances, such as reduction in response time $^{7}$ and vigilance to threat, ${ }^{8}$ and improved emotion recognition. ${ }^{9}$ However, there is insufficient evidence to state that tDCS has a positive or negative effect as a treatment for depression cognitive dysfunction ${ }^{10}$ and should therefore be further investigated.

Evidence of tDCS' efficacy on a depressive episode remains inconsistent, ${ }^{11,12}$ in part due to various confounding variables (ie, tDCS parameter settings, outcome measures, etc.) that are not under controlled. The effects of tDCS varying by individuals ${ }^{13}$ may also contribute to the heterogeneous results. The structural traits and functional states of individuals neural systems are considered the main sources of the individual sensitivity to tDCS. ${ }^{14}$ In addition, the baseline phenotypic predicts the response to tDCS. ${ }^{15}$ In other words, although several meta-analyses and systematic reviews suggest that the efficacy of tDCS is promising, ${ }^{11,12,16-18}$ the quality of the evidence is generally poor along with the unclear risk of bias. ${ }^{19}$ Therefore, it is necessary to further confirm its therapeutic efficacy and explore the predetermined factors of response to treatment through routine clinical and neuropsychological assessments.

\section{Transcranial Alternating Current Stimulation (tACS)}

TACS, another newly emerging neurological intervention, works by regularly oscillating electrical currents alternating between positive and negative voltages to modulate neural activity and phasis.

The alpha frequency $(8-12 \mathrm{~Hz})$ appears increased oscillatory activity in the frontal lobe of MDD patients, indicating that its regulation of mood, the processing of affective information, and emotional stimuli are disturbed $^{20}$. The increase in left hemisphere alpha activity is also associated with a lack of approach behavior. ${ }^{21}$

An RCT study found that 40 minutes $10 \mathrm{~Hz}$-tACS for 5 consecutive days can effectively target alpha oscillations in the frontal area, with its response rates significantly outperformed sham stimulation at the 2-week follow-up. Therefore, successful reshaping of disrupted oscillations may have changed the symptoms of MDD. ${ }^{22}$

The tACS may have potential antidepressant effects, but relevant RCTs are very limited, with only one study exploring the efficacy of $\mathrm{ACCS}^{22}$ and no studies comparing the effects of tACS with other common treatments.

\section{Safety of tDCS and tACS}

A meta-analysis has reported that low-intensity transcranial electrical stimulations, including tDCS and tACS, are safe. $^{23}$ TDCS was also well tolerated in the treatment of MDD and there was no significant difference in the rates of adverse event and drop-out between active and sham stimulation groups. ${ }^{24}$

Common adverse reactions to stimulation are itching, tingling sensation, burning sensation, headache, and discomfort at the stimulated region. Researchers can moisten the sponge by avoiding rubbing the skin where the electrodes stimulate, stimulating uneven (eg scarring) or inflamed skin areas, or using saline instead of tap water to reduce common adverse skin reactions. ${ }^{25}$ All these adverse events are brief and minor. ${ }^{23}$ Similar results are observed in tACS treatment. ${ }^{22}$ Active tES treatment strategies are at least as acceptable as sham treatment. ${ }^{19}$

Several studies have reported episodes of hypomania/ mania in patients with major depression after active tDCS stimulation. $^{26-28}$ And tDCS in combination with sertraline may cause hypomania/mania episodes. ${ }^{29}$ A meta-analysis revealed that even though the incidence of hypomania/mania episode was higher in the active stimulation group than in the sham group, the difference was not statistically significant. ${ }^{30}$ Overall, the causal relationship between tDCS and hypomania/mania episodes is difficult to prove given the low incidence and the limited number of subjects in a controlled trial. ${ }^{23}$

Previous studies often have some limitations, they include: (1) Small sample size; (2) Mixed samples with bipolar disorders or patients with treatment-resistant depression; (3) The stimulation protocol (stimulation target and time, current intensity, frequency, and the number of sessions as well as sham-stimulation) differs 
for one RCT to another; and (4) Fail to control other confounding factors, such as concurrent medications and psychotherapy, that may make it difficult to identify the main source of efficacy. As a review suggests medications that influence various neurotransmitter systems (GABA, dopamine, serotonin, etc.) may have an impact on tDCS effects on tissue excitability. ${ }^{31}$ Moreover, most of the previous evidence present a risk of low-quality or nebulous bias, ${ }^{19}$ which requires more precise estimates of the effect of treatment.

\section{Aims of the Study}

To verify the efficacy of tDCS and tACS, this study plans to conduct a randomized, double-blind, placebocontrolled clinical trial. We will compare the efficacy and safety of tDCS, tACS, sham stimulus/placebo, and one anti-depression drug, escitalopram. We choose the escitalopram because it shows relatively good efficacy and acceptability for the acute treatment of $\mathrm{MDD}^{32}$ and is commonly used in clinical practice in China. ${ }^{33-37}$ This drug should be adequate to be an active comparator as well as reflect real-world clinical practice. We will also identify the cognitive enhancement effect of tDCS and $\mathrm{tACS}$.

\section{Methods}

\section{Participants}

Participants are recruited by outpatient psychiatrists and advertisement posters in Shanghai Mental Health Center. Written informed consent is obtained from all the participants. Men and women age between 18 and 65 who meet the following criteria could be enrolled.

The inclusive criteria include:

1. Diagnosed with major depressive disorder (MDD) following DSM-5 (Diagnostic and Statistical Manual of Mental Disorders, 5th edition) criteria by psychiatrists. The diagnosis is conducted by Structured Clinical Interview (SCID) for DSM-V.

2. The presence of a depressive episode of at least moderate intensity, referring to Hamilton Depression Rating Scale (17-items) (HDRS-17) score $\geq 17$.

3. Participants with middle school and above education to ensure their ability to understand and complete the necessary measurements in the study;
Exclusive criteria:

1. Participants who meet the diagnostic criteria of other severe mental disorders including bipolar disorder, schizophrenia, alcohol and substance use disorders, personality and developmental disorders;

2. Patients with treatment-resistant depression (TRD), defined as who did not respond (eg, HDRS score reduction rate $<50 \%$ after treatment) to two successive antidepressant treatment of adequate dose and duration; ${ }^{38}$ patients using or who used escitalopram in the current acute depressive episode are also excluded because escitalopram is not comparable in such cases. However, those who used escitalopram in previous episodes and presented clinical responses can be included.

3. High risk of suicide and self-injury (ie score $\geq 3$ in the Hamilton suicide question);

4. Patients with the severe or unstable physical disease within 3 months or stroke within 30 days;

5. Pregnancy and lactation;

6. The subjects had attended any other clinical trials within 30 days before the baseline.

7. The subjects had taken any psychopharmacological drugs seven days before baseline. If the subjects are taking any pharmacological medication, there should be a wash-out period of at least 5 times the half-life of the drug. Specifically, a stable dosage of eszopiclone and zolpidem is allowed during the trial. Psychotherapy or any other systematic antidepressant treatment is not allowed during the screening and intervention phases.

Patients will withdraw from the study for the following reasons:

1. The patient requests for withdrawal for any reason;

2. The occurrence or deterioration of medical condition (eg, increased suicide risk, sudden onset of serious physical illness which would not allow the patient to continue the study, or participation in the study presents a significant burden to the patient);

3 . The occurrence of serious adverse events that cannot be tolerated by the patient,

4. Violation of the treatment protocol (eg, the patient requests for hospitalization or other approaches of treatment);

The reasons for withdrawal will be recorded factually in time. Participants who complete the study or 
withdraw from the study will be referred to an outpatient psychiatrist.

\section{Sample Size}

Since previous studies suggested that tDCS is more suitable for the initial treatment of depression, ${ }^{11}$ the response rate was chosen as the main endpoint measurement in this study. Our null hypothesis is that there is no significant difference in response rate between tDCS, tACS, Escitalopram, and sham/placebo groups. The study's primary aim is to prove the alternative hypothesis. Data for calculating the sample size for this study were obtained from comparable studies. The response rate for tACS, escitalopram, tDCS and sham/placebo is $77.8 \%,{ }^{22} 47 \%,{ }^{39}$ $41 \%{ }^{39}$ and $22 \%,{ }^{39}$ respectively. Power Analysis and Sample Size (PASS) software were used for a priori power analysis with a statistical power of $80 \%$, using a two-tailed test at the $5 \%$ level of significance, indicating that 88 subjects will be adequate to detect the difference in response rate. Taking 20\% drop-out rate into account, a sample size of 112 with 28 subjects in each group was determined.

\section{Intervention \\ Design}

Before the start of the study, we use a table of block random numbers generated by SPSS. Participants will be assigned to the corresponding intervention group according to their entry number (Please see Figure 1 for the study flow chart). The intervention groups include: active tDCS + placebo, active tACS + placebo, sham stimulus + escitalopram and sham stimulus + placebo. Physical

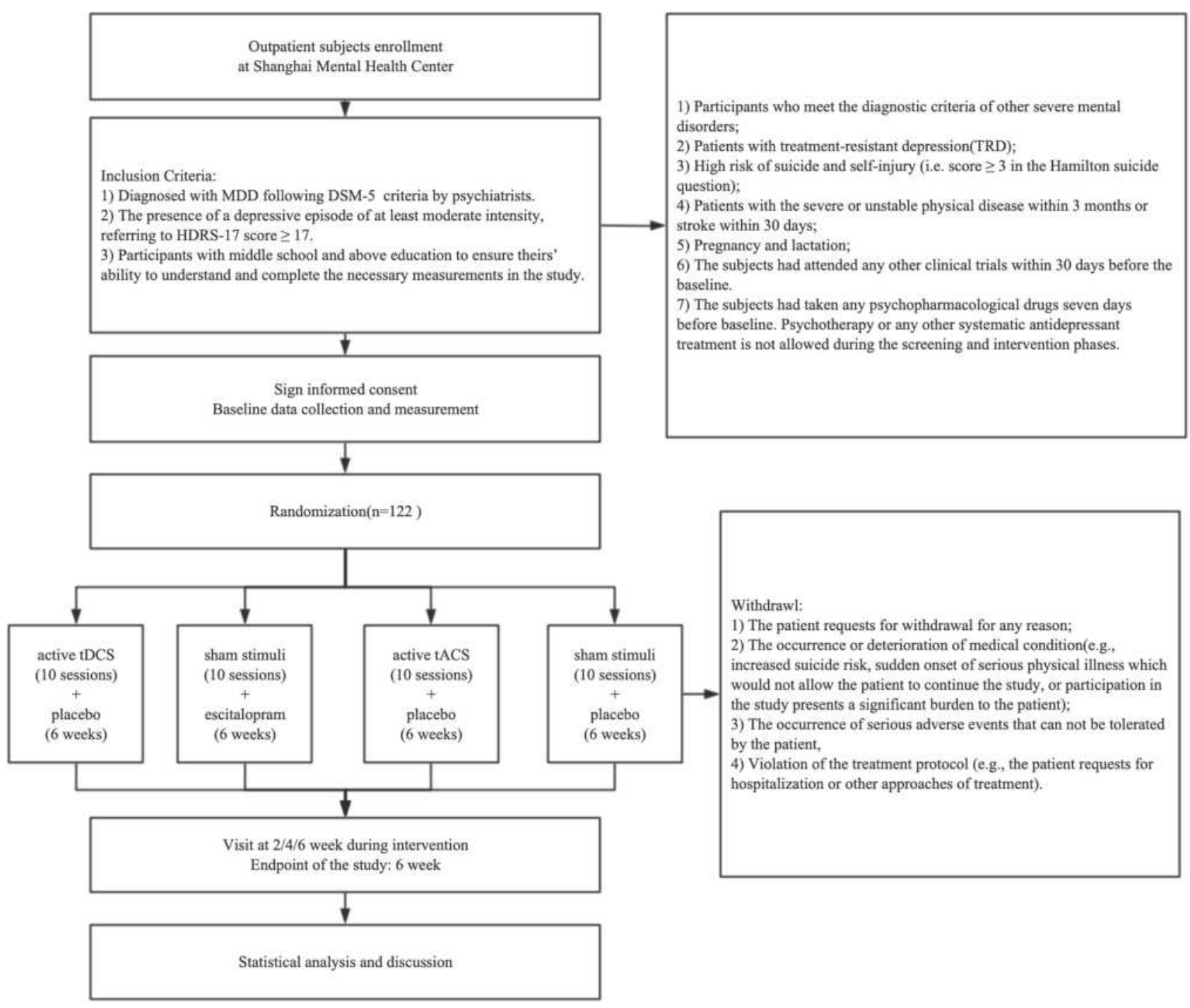

Figure I Study flow chart. 
stimulation starts at baseline and ends at week 2 , and medication starts at baseline and ends at week 6. During the last 4 weeks of our study, the pharmacological intervention alone, but without $\mathrm{tDCS} / \mathrm{tACS} /$ sham stimulations, was used and we wanted to observe if there was a delayed improvement in symptoms during this time, as previous studies have found that the behavioural effects of 20 to 30 minutes of tDCS stimulation lasted for approximately 90 minutes and the effects of 5 stimulation sessions were still detectable after 3 months. ${ }^{40}$

The visit windows are baseline, week 2, 4, and endpoint (week 6). There will be a clinical assessment at each visit. Cognitive assessments are at baseline and week 2. Functional imaging scanning is performed at baseline and week 6 . Safety assessments are at week 2 , 4 and 6. Measurement arrangements are shown in Table 1. Study measurement timetable

Table I The Study Measurement Schedule

\begin{tabular}{|c|c|c|c|c|}
\hline & Baseline & Week 2 & Week 4 & Week 6 \\
\hline \multicolumn{5}{|c|}{ Clinical Assessment } \\
\hline HDRS & $x$ & $x$ & $x$ & $x$ \\
\hline HAMA & $x$ & $x$ & $x$ & $x$ \\
\hline MADRS & $x$ & $x$ & $x$ & $x$ \\
\hline CGI & $x$ & $x$ & $x$ & $x$ \\
\hline YMRS & $x$ & $x$ & $x$ & $x$ \\
\hline ASRM & $x$ & $x$ & $x$ & $x$ \\
\hline $\begin{array}{l}\text { tDCS/tACS } \\
\text { adverse events }\end{array}$ & & $x$ & & \\
\hline SERS & & $x$ & $x$ & $x$ \\
\hline $\begin{array}{l}\text { Clinic lab } \\
\text { blood }\end{array}$ & $x$ & $x$ & $x$ & $x$ \\
\hline \multicolumn{5}{|c|}{ Cognitive Tests } \\
\hline WSCT & $x$ & $x$ & & \\
\hline $\begin{array}{l}\text { Stroop color- } \\
\text { word test }\end{array}$ & $x$ & $x$ & & \\
\hline \multicolumn{5}{|c|}{ Neurophysiological Measurements } \\
\hline EEG & $x$ & $x$ & & \\
\hline fMRI & $x$ & $x$ & & \\
\hline
\end{tabular}

Abbreviations: HDRS, Hamilton Depression Rating Scale; HAMA, Hamilton Anxiety Rating Scale; MADRS, Montgomery-Asberg Depression Rating Scale; CGI, Clinical Global Impression; YMRS, Young Mania Rating Scale; ASRM, Altman SelfRating Mania Scale; SERS, Asberg Side-effect Rating Scale for Antidepressant; WCST, Wisconsin Card Classification Test.

\section{Procedures}

The optimal protocol of tDCS (current intensity, stimulation target, duration, treatment frequency, etc.) is still being explored. The Canadian Network for Mood and Anxiety Treatments (CANMAT) 2016 clinical guidelines for the treatment of adult major depressive disorder recommended a minimum "dose" of tDCS stimulation: the current intensity of $2 \mathrm{~mA}$ for $30 \mathrm{~min} / \mathrm{d}$, once a day for a total of 10 times. ${ }^{41} \mathrm{In}$ this study, we follow the mentioned protocol. All stimulations are operated using the standard device (Starstim, Neuroelectrics Barcelona SL, Spain). The anode electrode is placed over the left dorsolateral prefrontal cortex (F3 in the International EEG System 10-20) and the cathode electrode over the right dorsolateral prefrontal cortex (F4). The electrodes are $5 \times 5 \mathrm{~cm}$ round sponges moistened with saline solution. There are 10 sessions for tDCS, tACS, and sham stimulation, with each session lasting for 30 minutes. To improve adherence and minimize dropouts, the study schedule is flexible, allowing participants to choose 10 days freely to receive intervention within 14 days.

For tDCS, the current intensity ramps up from 30 seconds to $2 \mathrm{~mA}$ and steadily lasts for 30 minutes, and ramps down to zero in the last 30 seconds. For tACS, one electrode serves as the anode while the other serves as the cathode during half cycle of an oscillation and then reverses at the peak intensity of $2 \mathrm{~mA}$. The sham group only receives the currents of $30 \mathrm{~s}$ rampup to $2 \mathrm{~mA}$ at the beginning of the session and $30 \mathrm{~s}$ ramp-down at the end, to mimic typical initial sensations of an active stimulus (eg, tingling, itching at the electrode sites) while minimizing potential neuromodulatory effects. ${ }^{42}$ The effect of this sham-protocol is similar to that of active stimulation, but less distinct. ${ }^{43}$

The escitalopram/placebo drugs are prescribed to the patient at baseline at a dosage of $10 \mathrm{mg} / \mathrm{d}$.

\section{Blinding}

Different stimulation protocols are pre-programmed on the computer by a dedicated person and the operator only follows the protocol codes, thus ensuring that neither the subject nor the operator is aware of the stimulation protocol being performed. Both escitalopram and placebo tablets are of the same appearance. The subjects and all study members involved in the enrolment, intervention operation, or assessment of participants are blinded to the study group. Blinding is also tested at the follow-up visit and endpoint of the study by asking subjects and raters to guess to which group they were assigned. 


\section{Ethic Issues}

The trial has registered in the Chinese Clinical Trial Registry (ChiCTR number: ChiCTR1800018063) on August 28, 2018. The study has been approved by the ethics committee at Shanghai Mental Health Center (registration approval number 2018-74R). Written informed consent is obtained from all participants. The study will comply with the Declaration of Helsinki.

\section{Outcome Measures}

\section{Clinical Assessment}

All clinical assessments will be administered by trained raters with established inter-rater reliability. In this study, categorical outcome (percentage of responders) is chosen as the primary outcome for it probably better reflects clinical practice. Response rate refers to the number of participants who achieved at least a $50 \%$ reduction in scores measured by the Montgomery-Asberg Depression Rating Scale (MADRS) compared to baseline. Specifically, participants who meet the criteria for clinical response at any of the 2, 4 and 6 week follow-up windows will be considered responders. We choose MARDS instead of HRDS as primary outcome measurement because the HRDS was not designed for use in intervention studies and should therefore only be used during screening visits as one of the inclusion criteria, whereas the MARDS is more sensitive to treatment effects and is more suitable as an indicator of efficacy. ${ }^{44}$ Secondary outcomes consist of clinical remission $(\mathrm{MARDS} \leq 10)$ rates and score change of other measurements, including Hamilton Anxiety Rating scale (HAMA), self-report Quick Inventory of Depressive Symptomatology (QIDS-SR) and Clinical Global Impression (CGI). ${ }^{45}$ All these measures have satisfactory psychometric properties in depression measurement. ${ }^{45,46}$

\section{Safety Assessment}

Safety is measured by a standard adverse event (AE) scale for $\mathrm{tES},{ }^{23}$ on which we record the frequency and severity of adverse events (eg headaches, tingling, itching, burning sensations, and any other phenomenon such as phosphenes). Asberg Side-effect Rating Scale for Antidepressant (SERS) is used to track adverse events of medications. Also, the Young Mania Rating Scale (YMRS) is used to monitor possible hypomanic and manic symptoms during the intervention. We also take blood samples as one of the safety indicators, mainly for monitoring Complete Blood Count (CBC) and liver and kidney function.

\section{Cognitive Assessment}

We chose two easily administered cognitive tests based on previous recommendations ${ }^{10}$ which are Wisconsin Card Sorting Test (WSCT) and Stroop test. WSCT is a neuropsychological test reflecting subjects' abstract generalization, working memory, cognitive transfer, and other aspects. ${ }^{47}$ Subjects are asked to sort 128 cards based on three possible categories (colors, number, and shape). After six consecutive correct responses, the sorting principle alerts us to another category. The test ends when subjects complete all six categories correctly or use all 128 cards. The preservative errors, the number of correct responses, the number of wrong responses, the total response time, and other of the total 13 indexes data before and after the intervention will be used for statistical analysis. Stroop Color-Word Test aims to test selective attention function and inhibition capacity and the performance of which improved significantly after antidepressant treatment. ${ }^{48} 120$ characters will be randomly presented with identical color (such as red "red"), contradictory color (such as green "red"), irrelevant color (such as green "middle"), irrelevant color semantics (such as blue "yellow") and neutral stimulus (color block), each stimulus presented for $1 \mathrm{~s}$. The subjects are asked to click on the corresponding color button for the four colors. The response time, the correct number, the number of errors, and the number of omissions are variables to be analyzed in this study.

\section{Functional MRI}

Functional magnetic resonance imaging (fMRI) will be carried out at baseline and endpoint (week 6). Functional MRIs are set as below. Siemens 3T magnetic resonance scanner is used to complete the scanning, including structure image, resting state, task state and DTI sequence. Structural image: scanning parameters are as follows: TR $=2500 \mathrm{~ms}, \quad \mathrm{TE}=3.5 \mathrm{~ms}$, TI $=1200 \mathrm{~ms}$, voxel size: $1.0 \times 1.0 \times 1.0 \mathrm{~mm}$, Flip angle $=8,45$ slices, FOV $=$ $256 \mathrm{~mm}$, Grappa $=2$; the whole scanning process lasts 5 minutes. Resting functional imaging: scanning parameters are as follows: $\mathrm{TR}=1400 \mathrm{~ms}, \mathrm{TE}=30 \mathrm{~ms}$, Flip angle $=80$, 64 slices, matrix $=112 \times 112, \mathrm{FOV}=224$, acquisition voxel size $=2.0 \times 2.0 \mathrm{~mm}$; the whole scanning process lasts 10 minutes. The subjects are given gaze points during the scanning, and all the subjects are required to keep their 
eyes open during the scanning process. Diffusion tensor imaging: scanning parameters are as follows: $\mathrm{TR}=$ $1400 \mathrm{~ms}, \mathrm{TE}=60 \mathrm{~ms}, 112 \times 112$ matrix, $\mathrm{FOV}=224 \mathrm{~mm}$, voxel size: $2.0 \times 2.0 \times 2.0 \mathrm{~mm}, \mathrm{~B} 1=1000,64$ slices, 137 non-collinear; the whole scanning process lasts 7 minutes.

We apply fMRI because it may provide critical information for response prediction ${ }^{14}$ and acts as an outcome measure of functional response to tDCS. Several studies have revealed the reductions of prefrontal gray matter volumes of the bilateral anterior cingulate cortices (ACC) and DLPFC in MDD. ${ }^{49-51}$ Gray matter volumes of the $\mathrm{ACC}^{51,52}$ and $\mathrm{PFC}^{51,53,54}$ at baseline predicted treatment response. And the volumes of these regions increased after successful antidepressant treatment. ${ }^{5,56}$ Therefore, we will analyze voxel-based gray matter volumes of PFC and ACC region at baseline and endpoint using parcellation approaches.

\section{Statistical Analysis}

The statistician will analyze the information collected in an intention-to-treat (ITT) dataset and impute missing follow-up observation caused by poor compliance using the last observation carried forward (LOCF) design because missing data are presented to be minimal and thus can be considered at random in similar study. ${ }^{27,57}$ We will also compare the baseline characteristics of participants with missing follow-up data and those without missing data using ANOVA, $\chi 2$ or Kruskal-Wallis test for continuous variables and categorical variables, respectively. Although our main endpoint is response rates, the remission rates will be presented too.

Multiple comparison issues of post hoc analysis will be carried out using Bonferroni adjustment. Specifically, although in our speculation, these variables would not be normally distributed, we will apply parametric tests because the Central Limit Theorem states that this approach in more than 30 observations is permitted. ${ }^{58}$ The Fisher's exact test will be used to compare the frequency of adverse events in each group. A mixed ANOVA will be performed with one dependent within-subject variable (eg, score change of MADRS and HRDS), one independent within-subject variable (visit time - four levels), and one independent between-subject variable (group four levels). General linear models will also be constructed to identify whether the demographics, descriptive and cognitive test variables predict individual response to tDCS. A two-sided 5\% significance level will be considered statistically significant.

\section{Discussion}

To the best of our knowledge, the current study is the first parallel double-blind placebo-controlled trial exploring tACS, tDCS, and escitalopram in the treatment of MDD. Confounding factors such as concurrent medication, psychotherapy, and other treatment approaches are strictly controlled to probe the pure effect of tDCS and tACS. Considering that our sample size is not large enough to enroll an excessively heterogeneous sample, we also exclude participants with treatment-resistant depression (TRD), as TRD has been established as a negative predictor of treatment response. ${ }^{11,18,27,28,59,60}$ In general, we expect patients with ongoing, moderate to the severe depressive episodes with a history of treatment of only 1-2 medications or no previous medication. This study is currently being conducted. Ideally, we will complete and conclude the study within the original sample size plan and within the timeframe of the grant.

There were only two previous studies that compared tDCS with antidepressant drugs and were both conducted in the cultural context of Latin America. Previous research has found that active tDCS $(2 \mathrm{~mA}, 30 \mathrm{~min}, 12$ sessions $)$ and $50 \mathrm{mg} / \mathrm{d}$ sertraline had similar efficacy over 6 weeks, ${ }^{28}$ while one other study presented that tDCS (30-minute, 2-mA, 15 sessions) did not show non-inferiority to escitalopram over a 10 -week period. ${ }^{39}$ It should be noted that depression symptoms vary from cultures and requires various treatment approaches, for example, somatization is more severe and common in China. ${ }^{61-63}$ It is necessary to provide more evidence of tDCS and tACS treating MDD in the cultural context of China.

The limitations of this trial are the relatively short duration of the trial (6 weeks) and the low dose of escitalopram $(10 \mathrm{mg} / \mathrm{d})$ or active tDCS ( $2 \mathrm{~mA}, 30$ minutes, 10 sessions $)$, as symptoms may improve over 2 to 3 months. ${ }^{32}$ And we failed to combined active tDCS/tACS with medications, which is a promising field since the former study has found the sertraline combined with tDCS shows significantly better efficacy than tDCS only or sertraline only. ${ }^{27}$ These limitations are the result of financial and feasibility constraints. Also, our trial lacked a blank control or waiting group. As $\mathrm{tDCS} / \mathrm{tACS}$ is a new type of treatment, the research staff will take a relatively long time to explain the procedure to the patient and obtain informed consent. During the first two weeks of intervention, patients return to the hospital daily. The raters will follow a patient for 6 weeks and there will be frequent contact (eg to make appointments to return to the 
hospital or to answer questions from the patient). Active participation in the experiment itself can have a therapeutic effect because patients may develop a good doctor-patient relationship with friendly researchers, have time to talk and feel supported by the researcher during the engagement process. ${ }^{64}$ These may result in confounding factors for evaluating the effectiveness of the treatment. Another limitation of the trial is that we are unable to confirm the physiological effects of sham-stimulation using EEG due to various limitations, since the sham stimulation itself may have enhanced effects due to the cumulative effect of repeated delivery. $^{65}$

In summary, we expect the results of our trial to provide more evidence that may potentially advance our advances in physical stimulation intervention and benefit clinical practice in the treatment of MDD. These would include: 1) whether the non-invasive physical therapy interventions (tDCS) and (tACS) could be a safe and useful intervention for MDD; 2) Any difference of efficacy between tDCS and tACS in treating MDD; 3) comparing to medications, whether the tDCS and tACS may have comparable efficacy in symptom improvement; and 4) whether the treatment of tDCS or tACS would also improve the cognitive function in MDD.

\section{Funding}

The study was funded by the Municipal Hospital Clinical Research Cultivating Project of Shanghai Shenkang Hospital Development Center(SHDC12018X24).

\section{Disclosure}

The authors report no conflicts of interest in this work.

\section{References}

1. Huang Y, Wang Y, Wang H, et al. Prevalence of mental disorders in China: a cross-sectional epidemiological study. Lancet Psychiatry. 2019;6(3):211-224. doi:10.1016/S2215-0366(18)30511-X

2. Rush AJ. Limitations in efficacy of antidepressant monotherapy. J Clin Psychiatry. 2007;68(10):8-10.

3. John Rush A, Trivedi MH, Wisniewski SR, et al. Acute and longerterm outcomes in depressed outpatients requiring one or several treatment steps: a STAR*D report. $J$ Am $J$ Psychiatry. 2006;163 (11):1905-1917. doi:10.1176/ajp.2006.163.11.1905

4. Brunoni AR, Boggio PS, Ferrucci R, Priori A, Fregni F. Transcranial direct current stimulation: challenges, opportunities, and impact on psychiatry and neurorehabilitation. Front Psychiatry. 2013;4:19. doi:10.3389/fpsyt.2013.00019

5. Yamada Y, Sumiyoshi T. Neurobiological mechanisms of transcranial direct current stimulation for psychiatric disorders; neurophysiological, chemical, and anatomical considerations. Front Hum Neurosci. 2021;15:631838. doi:10.3389/fnhum.2021.631838
6. Lefaucheur JP, Antal A, Ayache SS, et al. Evidence-based guidelines on the therapeutic use of transcranial direct current stimulation (tDCS). Clin Neurophysiol. 2017;128(1):56-92.

7. Brunoni AR, Vanderhasselt MA. Working memory improvement with non-invasive brain stimulation of the dorsolateral prefrontal cortex: a systematic review and meta-analysis. Brain Cogn. 2014;86:1-9. doi:10.1016/j.bandc.2014.01.008

8. Ironside M, O'Shea J, Cowen PJ, Harmer CJ. Frontal cortex stimulation reduces vigilance to threat: implications for the treatment of depression and anxiety. Biol Psychiatry. 2016;79(10):823-830. doi:10.1016/j.biopsych.2015.06.012

9. Brennan S, Mcloughlin DM, O'Connell R, et al. Anodal transcranial direct current stimulation of the left dorsolateral prefrontal cortex enhances emotion recognition in depressed patients and controls. $J$ Clin Exp Neuropsychol. 2017;39(4):1-12. doi:10.1080/ 13803395.2016.1230595

10. Brunoni AR, Tortella G, Bensenor IM, Lotufo PA, Carvalho AF, Fregni F. Cognitive effects of transcranial direct current stimulation in depression: results from the SELECT-TDCS trial and insights for further clinical trials. $J$ Affect Disord. 2016;202:46-52.

11. Brunoni AR, Moffa AH, Fregni F, et al. Transcranial direct current stimulation for acute major depressive episodes: meta-analysis of individual patient data. British $j$ Psychiatry. 2016;208(6):522-531. doi:10.1192/bjp.bp.115.164715

12. Shiozawa P, Fregni F, Benseñor IM, et al. Transcranial direct current stimulation for major depression: an updated systematic review and meta-analysis. Int J Neuropsychopharmacol. 2014;17(9):1443-1452. doi:10.1017/S1461145714000418

13. Chew T, Ho KA, Loo CK. Inter- and intra-individual variability in response to transcranial direct current stimulation (tDCS) at varying current intensities. Brain Stimul. 2015;8(6):1130-1137. doi:10.1016/j. brs.2015.07.031

14. Esmaeilpour Z, Shereen AD, Ghobadi-Azbari P, et al. Methodology for tDCS integration with fMRI. Hum Brain Mapp. 2020;41 (7):1950-1967. doi:10.1002/hbm.24908

15. Kambeitz J, Goerigk S, Gattaz W, et al. Clinical patterns differentially predict response to transcranial direct current stimulation (tDCS) and escitalopram in major depression: a machine learning analysis of the ELECT-TDCS study. $J$ Affect Disorders. 2020;265:460-467. doi:10.1016/j.jad.2020.01.118

16. Fregni F, El-Hagrassy MM, Pacheco-Barrios K, et al. Evidence-based guidelines and secondary meta-analysis for the use of transcranial direct current stimulation (tDCS) in neurological and psychiatric disorders. Int $j$ Neuropsychopharmacol. 2020.

17. Meron D, Hedger N, Garner M, Baldwin DS. Transcranial direct current stimulation (tDCS) in the treatment of depression: systematic review and meta-analysis of efficacy and tolerability. Neurosci Biobehav Rev. 2015;57:46-62. doi:10.1016/j.neubiorev.2015.07.012

18. Mutz J, Edgcumbe DR, Brunonimd AR, Fumd CHYF. Efficacy and acceptability of non-invasive brain stimulation for the treatment of adult unipolar and bipolar depression: a systematic review and meta-analysis of randomised sham-controlled trials. Neurosci Biobehav Rev. 2018;92:291-303. doi:10.1016/j. neubiorev.2018.05.015

19. Mutz J, Vipulananthan V, Carter B, Hurlemann R, Fu CHY, Young AH. Comparative efficacy and acceptability of non-surgical brain stimulation for the acute treatment of major depressive episodes in adults: systematic review and network meta-analysis. BMJ (Clinical Research Ed). 2019;364:11079. doi:10.1136/bmj.11079

20. Leuchter AF, Cook IA, Hunter AM, Cai C, Horvath S. Resting-state quantitative electroencephalography reveals increased neurophysiologic connectivity in depression. PLoS One. 2012;7(2):e32508. doi: 10.1371 journal.pone. 0032508

21. de Aguiar Neto FS, Rosa JLG. Depression biomarkers using non-invasive EEG: a review. Neurosci Biobehav Rev. 2019;105:83-93. doi:10.1016/j.neubiorev.2019.07.021 
22. Alexander ML, Alagapan S, Lugo CE, et al. Double-blind, randomized pilot clinical trial targeting alpha oscillations with transcranial alternating current stimulation (tACS) for the treatment of major depressive disorder (MDD). Transl Psychiatry. 2019;9(1):106. doi:10.1038/s41398-019-0439-0

23. Antal A, Alekseichuk I, Bikson M, et al. Low intensity transcranial electric stimulation: safety, ethical, legal regulatory and application guidelines. Clin Neurophysiol. 2017;128(9):1774-1809.

24. Moffa AH, Brunoni AR, Fregni F, et al. Safety and acceptability of transcranial direct current stimulation for the acute treatment of major depressive episodes: analysis of individual patient data. $J$ Affect Disord. 2017;221:1-5. doi:10.1016/j.jad.2017.06.021

25. Palm U, Schiller C, Fintescu Z, et al. Transcranial direct current stimulation in treatment resistant depression: a randomized double-blind, placebo-controlled study. Brain Stimul. 2012;5 (3):242-251. doi:10.1016/j.brs.2011.08.005

26. Li MS, Chen YY, Hung GC. Rapid onset of hypomania associated with transcranial direct current stimulation in a patient with bipolar depression. Brain Stimul. 2016;9(6):955-956. doi:10.1016/j. brs.2016.08.008

27. Brunoni AR, Valiengo L, Baccaro A, et al. The sertraline vs electrical current therapy for treating depression clinical study results from a factorial, randomized, controlled trial. JAMA Psychiatry. 2013;70 (4):383-391. doi:10.1001/2013.jamapsychiatry.32

28. Valiengo L, Bensenor IM, Goulart AC, et al. The sertraline versus electrical current therapy for treating depression clinical study (select-TDCS): results of the crossover and follow-up phases. Depress Anxiety. 2013;30(7):646-653. doi:10.1002/da.22079

29. Brunoni AR, Valiengo L, Zanao T, de Oliveira JF, Bensenor IM, Fregni F. Manic psychosis after sertraline and transcranial direct-current stimulation. $J$ Neuropsychiatry Clin Neurosci. 2011;23(3):E4-5. doi:10.1176/jnp.23.3.jnpe4

30. Brunoni AR, Moffa AH, Sampaio-Júnior B, Gálvez V, Loo CK. Treatment-emergent mania/hypomania during antidepressant treatment with transcranial direct current stimulation (tDCS): a systematic review and meta-analysis. Brain Stimul. 2017;10 (2):260-262. doi:10.1016/j.brs.2016.11.005

31. McLaren ME, Nissim NR, Woods AJ. The effects of medication use in transcranial direct current stimulation: a brief review. Brain Stimul. 2018;11(1):52-58. doi:10.1016/j.brs.2017.10.006

32. Cipriani A, Furukawa TA, Salanti G, et al. Comparative efficacy and acceptability of 21 antidepressant drugs for the acute treatment of adults with major depressive disorder: a systematic review and network meta-analysis. Lancet. 2018;391(10128):1357-1366. doi:10.1016/S0140-6736(17)32802-7

33. Zhong C, Zeng H, Zhang X, Li Y. Application of antidepressant drugs in a hospital from 2014 to 2016. China Pharm. 2018;27:75-78.

34. Wang L, Wu F. Analysis of the utilization of antidepressant drugs in 11 hospitals from Zhejiang province during 2013-2017. China Pharmacy. 2019;30(05):134-137.

35. Qin J, Zhang Y, Liang Y. Antidepressants use in Chinese PLA General Hospital from 2012 to 2016. Acad J Chin Pla Med School. 2017;038(8):P.802-806.

36. Pan H, Liu H, Xu J, Wang J. Application of antidepressive drugs used in 31 hospitals of Nanjing area during 2013 to 2015. China Pharmacy. 2016;35.

37. Guo C, Zhang Y, Qiao Z, Du W. Analysis of antidepressant drugs used in our hospital from 2014 to 2016. China Pharm. 2017;12.

38. McIntyre RS, Filteau M-J, Martin L, et al. Treatment-resistant depression: definitions, review of the evidence, and algorithmic approach. $J$ Affect Disorders. 2014;156:1-7. doi:10.1016/j. jad.2013.10.043

39. Brunoni AR, Moffa AH, Sampaio-Junior B, et al. Trial of electrical direct-current therapy versus escitalopram for depression. $N$ Engl J Med. 2017;376(26):2523-2533. doi:10.1056/NEJMoa1612999
40. Ww F, Bm G, S K. Review of transcranial direct current stimulation in poststroke recovery. Top Stroke Rehabil. 2013;20:68-77. doi:10.1310/tsr2001-68

41. Milev RV, Giacobbe P, Kennedy SH, et al. Canadian Network for Mood and Anxiety Treatments (CANMAT) 2016 clinical guidelines for the management of adults with major depressive disorder: section 4. Neurostimulation Treatments. Can J Psychiatry. 2016;61 (9):561-575. doi:10.1177/0706743716660033

42. Loo CK, Husain MM, McDonald WM, et al. International randomized-controlled trial of transcranial Direct Current Stimulation in depression. Brain Stimul. 2018;11(1):125-133. doi:10.1016/j.brs.2017.10.011

43. Boonstra TW, Nikolin S, Meisener A-C, Martin DM, Loo CK. Change in mean frequency of resting-state electroencephalography after transcranial direct current stimulation. Front Hum Neurosci. 2016;10:270. doi:10.3389/fnhum.2016.00270

44. Bajbouj M, Aust S, Spies J, et al. PsychotherapyPlus: augmentation of cognitive behavioral therapy (CBT) with prefrontal transcranial direct current stimulation (tDCS) in major depressive disorder-study design and methodology of a multicenter double-blind randomized placebo-controlled trial. Eur Arch Psychiatry Clin Neurosci. 2018;268(8):797-808. doi:10.1007/s00406-017-0859-x

45. de Beurs E, Carlier IVE, van Hemert AM. Approaches to denote treatment outcome: clinical significance and clinical global impression compared. Int J Methods Psychiatr Res. 2019;28(4):e1797. doi:10.1002/mpr.1797

46. Bernstein IH, Rush AJ, Stegman D, Macleod L, Witte B, Trivedi MHA. Comparison of the QIDS-C16, QIDS-SR16, and the MADRS in an Adult Outpatient Clinical Sample. CNS Spectr. 2010;15(7):458-468. doi:10.1017/S1092852900000389

47. Berg EA. A simple objective technique for measuring flexibility in thinking. $J$ Gen Psychol. 1948;39:15-22. doi:10.1080/ 00221309.1948.9918159

48. Wagner S, Doering B, Helmreich I, Lieb K, Tadić A. A meta-analysis of executive dysfunctions in unipolar major depressive disorder without psychotic symptoms and their changes during antidepressant treatment. Acta Psychiatr Scand. 2012;125(4):281-292. doi:10.1111/ j.1600-0447.2011.01762.x

49. Lai CH. Gray matter volume in major depressive disorder: a meta-analysis of voxel-based morphometry studies. Psychiatry Res. 2013;211(1):37-46. doi:10.1016/j.pscychresns.2012. 06.006

50. Bora E, Fornito A, Pantelis C, Yucel M. Gray matter abnormalities in Major Depressive Disorder: a meta-analysis of voxel based morphometry studies. J Affect Disord. 2012;138(1-2):9-18. doi:10.1016/j. jad.2011.03.049

51. Fonseka TM. Neuroimaging biomarkers as predictors of treatment outcome in Major Depressive Disorder. J Affect Disorders. 2018;233 (1):21-35. doi:10.1016/j.jad.2017.10.049

52. Wise T, Radua J, Via E, et al. Common and distinct patterns of grey-matter volume alteration in major depression and bipolar disorder: evidence from voxel-based meta-analysis. Mol Psychiatry. 2017;22(10):1455-1463. doi:10.1038/mp.2016.72

53. Costafreda SG, Chu C, Ashburner J, Fu CH. Prognostic and diagnostic potential of the structural neuroanatomy of depression. PLoS One. 2009;4(7):e6353. doi:10.1371/journal. pone. 0006353

54. Bulubas L, Padberg F, Bueno PV, et al. Antidepressant effects of tDCS are associated with prefrontal gray matter volumes at baseline: evidence from the ELECT-TDCS trial. Brain Stimul. 2019;12 (5):1197-1204. doi:10.1016/j.brs.2019.05.006

55. Fu CH, Costafreda SG, Sankar A, et al. Multimodal functional and structural neuroimaging investigation of major depressive disorder following treatment with duloxetine. BMC Psychiatry. 2015;15:82. doi:10.1186/s12888-015-0457-2 
56. Smith R, Chen K, Baxter L, Fort C, Lane RD. Antidepressant effects of sertraline associated with volume increases in dorsolateral prefrontal cortex. J Affect Disord. 2013;146(3):414-419. doi:10.1016/j. jad.2012.07.029

57. Brunoni AR, Valiengo L, Baccaro A, et al. Sertraline vs. ELectrical Current Therapy for Treating Depression Clinical Trial-SELECT TDCS: design, rationale and objectives. Contemp Clin Trials. 2011;32(1):90-98. doi:10.1016/j.cct.2010.09.007

58. Fortney L. Foundations of clinical research: applications to practice. Connecticut Appleton Lange. 2013;53.

59. Bennabi D, Nicolier M, Monnin J, et al. Pilot study of feasibility of the effect of treatment with tDCS in patients suffering from treatment-resistant depression treated with escitalopram. Clin Neurophysiol. 2015;126(6):1185-1189. doi:10.1016/j.clinph.2014.09.026

60. Palm U, Hasan A, Strube W, Padberg F. tDCS for the treatment of depression: a comprehensive review. Eur Arch Psychiatry Clin Neurosci. 2016;266(8):681-694. doi:10.1007/s00406-016-0674-9

61. Dreher A, Hahn E, Diefenbacher A, et al. Cultural differences in symptom representation for depression and somatization measured by the PHQ between Vietnamese and German psychiatric outpatients. J Psychosom Res. 2017;102:71-77. doi:10.1016/j. jpsychores.2017.09.010
62. Jeon HJ, Walker RS, Inamori A, et al. Differences in depressive symptoms between Korean and American outpatients with major depressive disorder. Int Clin Psychopharmacol. 2014;29 (3):150-156. doi:10.1097/YIC.0000000000000019

63. Ryder AG, Chentsova-Dutton YE. Depression in cultural context: "Chinese somatization," revisited. Psychiatr Clin North Am. 2012;35(1):15-36. doi:10.1016/j.psc.2011.11.006

64. Tallon D, Mulligan J, Wiles N, et al. Involving patients with depression in research: survey of patients' attitudes to participation. $\mathrm{Br}$ J Gen Pract. 2011;61(585):134-141. doi:10.3399/bjgp11X567036

65. Fonteneau C, Mondino M, Arns M, et al. Sham tDCS: a hidden source of variability? Reflections for further blinded, controlled trials. Brain Stimul. 2019;12:668-673. doi:10.1016/j.brs.2018.12.977

\section{Publish your work in this journal}

Neuropsychiatric Disease and Treatment is an international, peerreviewed journal of clinical therapeutics and pharmacology focusing on concise rapid reporting of clinical or pre-clinical studies on a range of neuropsychiatric and neurological disorders. This journal is indexed on PubMed Central, the 'PsycINFO' database and CAS, and is the official journal of The International Neuropsychiatric Association (INA). The manuscript management system is completely online and includes a very quick and fair peer-review system, which is all easy to use. Visit http://www.dovepress.com/testimonials.php to read real quotes from published authors. 